\title{
Simulation of Composite Particle Growth in the Dispersion and Compounding Processes of Alloy Particles and Ceramic Powder ${ }^{\dagger}$
}

\author{
Tomohiro Iwasaki, Takeshi Yanagida \\ and Munetake Satoh \\ Dept. of Chem. Eng., Osaka Pref. Univ.*
}

\begin{abstract}
The authors investigated a compounding process in which fine WC powder is uniformly dispersed into particulate Ag-Ni alloy with a high-speed elliptical-rotor-type powder mixer. The growth of composite particles is observed as processing time elapses. A model for the growth phenomenon is constructed on the basis of the theory of powder grinding with a ball mill, and of the dynamics of the plastic deformation of metal particles. In the model, a function of the probability that composite particles grow is expressed in terms of three factors that include the compounding conditions as parameters: (1) the probability that particles are caught between a pair of medium balls (zirconia beads), (2) that they have undergone plastic deformation, and (3) the frequency with which the mixture of composite particles and beads is compressed by the elliptical rotor at the minimum clearance per unit time. Temporal change in the size distribution of composite particles has been calculated by using model equations. Comparison of calculated values with the experimental data for particle median diameter shows that they agree closely. It was demonstrated that the model is valid and that it is possible to accurately estimate and control particle size.
\end{abstract}

\section{Introduction}

In the powder processing in which the particle size changes, such as granulation, comminution, and others, formulating the size distribution of products in time series makes it possible to control the products' physical properties and estimate the processing time in which products of a required state are obtained. Recently in the field of powder metallurgy there has been active study of mechanical alloying (MA), mechanical grinding $(\mathrm{MG})$, and other processes in which materials are compounded mechanically. However, there has been little research analyzing temporal changes in the size of composite particles on the basis of kinetics.

The authors have evaluated processing in which fine ceramic powder (tungsten carbide) is dispersed uniformly into a particulate alloy of silver and nickel with a high-speed elliptical-rotor-type powder mixer. Effects of compounding conditions on the state of the

* 1-1 Gakuen-cho, Sakai, Osaka 599-8531, Japan TEL. 0722-54-9307 FAX. 0722-54-9911

+ This report was originally printed in J. of Japan Soc. of Powder and Powder Metallurgy, 45, No. 4 362-367 (1998) in Japanese, before being translated into English by KONA Editorial Committee with the permission of the editorial committee of the Japan Society of Powder and Powder Metallurgy. composite particles obtained were investigated by measuring the temporal change in the lightness and size of composite particles. ${ }^{1,2}$ A simplified model of chemical reaction between two species was applied to the growth phenomenon of composite particles as time passed during the process. It was found that experimental conditions, such as the volume ratio of the powder mixture to the medium (zirconia beads) and the amount of ceramic powder added, had a great qualitative influence on the rate of particle growth and the degree of compounding. ${ }^{2}$

In this paper, a kinetics model of particle growth has been constructed on the basis of the theory of powder grinding with a ball mill ${ }^{3}$ and of the dynamics of the plastic deformation of metal particles. This model takes into account the operating conditions of the powder mixer, the physical properties of powders, and the effects of ceramic powder in composite particles on plastic deformation. Temporal changes in the size distribution of composite particles have been simulated in terms of the model equations.

\section{Model of particle growth}

\subsection{Kinetics model}

The frequency distribution function $f\left(D_{\mathrm{p}}, t\right)$ of the composite particles with diameter $D_{\mathrm{p}}$ (area basis) at 
processing time $t$ is derived from the population balance as follows:

$$
\frac{\mathrm{d}}{\mathrm{d} t}\left[S(t) \cdot f\left(D_{\mathrm{p}}, t\right) \cdot \mathrm{d} D_{\mathrm{p}}\right]=S_{\text {in }}-S_{\text {out }}
$$

where $S(t)$ indicates the total projected area of composite particles. $S_{\text {in }}$ and $S_{\text {out }}$ are the projected areas of the composite particles, the sizes of which are within and without the size range between $D_{\mathrm{p}}$ and $D_{\mathrm{p}}+\mathrm{d} D_{\mathrm{p}}$ per unit time, respectively.

Observations of composite particles with a scanning electron microscope confirmed that composite particles were discoidal and that their thickness was uniform in the compounding process. ${ }^{2}$ We proposed a simplified model for the particle growth phenomenon of the process. As illustrated in Figure 1, the model indicates that composite particles grow in the following manner: (1) a pair of colliding beads holds two disk-shaped particles (diameter $D_{\mathrm{p}} / \sqrt{2}$ and thickness h) and sticks the particles together, and (2) a particle of diameter $D_{\mathrm{p}}$ (particle density and thickness are same as those of the particles before growth) is newly formed by the fused particles, causing plastic deformation. $S_{\text {in }}$ and $S_{\text {out }}$ are given by Eqs. (2) and (3):

$$
\begin{aligned}
& S_{\text {in }}=P_{z}\left(D_{\mathrm{p}} / \sqrt{2}\right) \cdot S(t) \cdot f\left(D_{\mathrm{p}} / \sqrt{2}, t\right) \cdot \mathrm{d} D_{\mathrm{p}} \\
& S_{\text {out }}=P_{z}\left(D_{\mathrm{p}}\right) \cdot S(t) \cdot f\left(D_{\mathrm{p}}, t\right) \cdot \mathrm{d} D_{\mathrm{p}}
\end{aligned}
$$

where $P_{z}\left(D_{\mathrm{p}}\right)$ is a function of the probability that a particle of diameter $D_{\mathrm{p}}$ will grow per unit time.

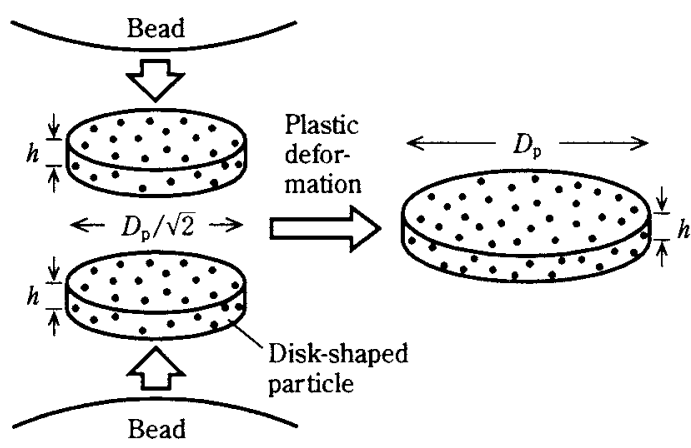

Fig. 1 Compounding model for disk-shaped particles

\subsection{Theoretical analysis of the probability func- tion $\boldsymbol{P}_{\mathbf{z}}\left(D_{\mathrm{p}}\right)$ of particle growth}

The probability function $P_{\mathrm{z}}\left(D_{\mathrm{p}}\right)$ of particle growth is defined as the product of a function $P_{\mathrm{n}}\left(D_{\mathrm{p}}\right)$ of the probability that two particles are held between a pair of beads, $P_{\mathrm{pl}}\left(D_{\mathrm{p}}\right)$ that plastic deformation occurs, and the frequency $Z$ with which the mixture of composite particles and beads is compressed by the rotor at the minimum clearance per unit time.

$$
P_{z}\left(D_{\mathrm{p}}\right)=P_{\mathrm{n}}\left(D_{\mathrm{p}}\right) \cdot P_{\mathrm{pl}}\left(D_{\mathrm{p}}\right) \cdot Z
$$

$Z$ is given by

$$
Z=2\left(N_{\mathrm{r}}+N_{\mathrm{v}}\right)
$$

where $N_{\mathrm{r}}$ and $N_{\mathrm{v}}$ are the rotational speeds of the rotor and the vessel, respectively.

\section{Probability of holding composite particles}

The apparent volume $V_{\mathbf{a}}$ of the mixture of metal particles and ceramic powder in the vessel is expressed on the basis of the charging condition of the powder mixture and the beads as follows:

$$
V_{\mathrm{a}}=V_{\mathrm{m}} f \frac{V_{\mathrm{p}} / V_{\mathrm{b}}}{1+V_{\mathrm{p}} / V_{\mathrm{b}}}
$$

$V_{\mathrm{m}}$ is the effective volume of the vessel, and $f$ is the charge volume ratio. $V_{\mathrm{p}}$ and $V_{\mathrm{b}}$ are the apparent charge volumes of the powder mixture and the beads, respectively.

By assuming that two composite particles held between a pair of beads adhere rapidly to each other and then form one particle in appearance, the apparent volume $v_{\mathrm{a}}$ of the composite particles existing in voids among the beads is expressed geometrically (see Figure 2).

$$
\begin{aligned}
& v_{\mathrm{a}}=\pi b^{2} D_{\mathrm{p}} \\
& b^{2} \cong \frac{1}{2} D_{\mathrm{p}} D_{\mathrm{b}}
\end{aligned}
$$

where $D_{\mathrm{p}}$ and $D_{\mathrm{b}}$ are the diameters of the composite particle and the bead, respectively.

As illustrated in Figure 2, the forces $\mu F \cos ^{2} \alpha$ and $F \cos \alpha \sin \alpha$ act simultaneously on the composite particles held between a pair of colliding beads: the former is the force by which composite particles are pulled in between the beads, and the latter repels the particles. $F$ is the impact force of the beads and $\mu$ is coefficient of dynamic friction. The composite particles are

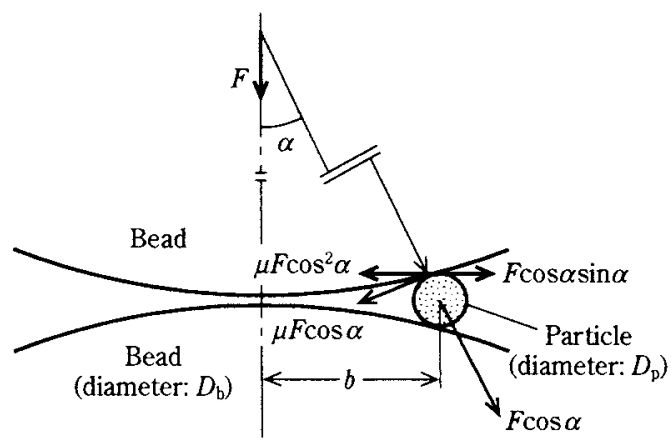

Fig. 2 Schematic diagram of a composite particle held between a pair of beads 
caught by the beads when the following condition is satisfied:

$$
\begin{aligned}
& \mu F \cos ^{2} \alpha \geq F \cos \alpha \sin \alpha \\
& \therefore \tan \alpha \leq \mu
\end{aligned}
$$

The area ratio $\gamma$ of the region where the composite particles are held and that where the forces act upon them is expressed by Eq. (11) in terms of $\xi=\tan ^{-1} \mu$.

$$
\gamma=\frac{\left[\left(D_{\mathrm{b}} / 2\right) \tan \xi\right]^{2}}{b^{2}}=\frac{\mu^{2}}{2}\left(\frac{D_{\mathrm{b}}}{D_{\mathrm{p}}}\right)
$$

Therefore, $P_{\mathrm{n}}\left(D_{\mathrm{p}}\right)$ is expressed by Eq. (12).

$$
P_{\mathrm{n}}\left(D_{\mathrm{p}}\right)=\frac{v_{\mathrm{a}}}{V_{\mathrm{a}}} \times \gamma=\frac{\pi \mu^{2} D_{\mathrm{p}} D_{\mathrm{b}}^{2}}{4 V_{\mathrm{m}} f}\left(\frac{V_{\mathrm{p}} / V_{\mathrm{b}}}{1+V_{\mathrm{p}} / V_{\mathrm{b}}}\right)^{-1}
$$

In consideration of the shape of composite particles in the compounding process, Eq. (12) is corrected with the shape factor $\phi$.

$$
P_{\mathrm{n}}\left(D_{\mathrm{p}}\right)=\frac{\pi \mu^{2} D_{\mathrm{p}} D_{\mathrm{b}}^{2}}{4 V_{\mathrm{m}} f}\left(\frac{V_{\mathrm{p}} / V_{\mathrm{b}}}{1+V_{\mathrm{p}} / V_{\mathrm{b}}}\right)^{-\phi}
$$

$\phi$ is defined by the following equation:

$$
\phi=A / A_{\mathrm{s}}
$$

where $A$ and $A_{\mathrm{s}}$ indicate the projected areas of composite particles and spherical particles, respectively, having volumes equal to that of the composite particle.

\section{Probability of plastic deformation occurring}

From the equilibrium of stress in the radial direction in the disk-shaped particle as shown in Figure 3, the following equation is obtained in terms of the approximation $\sin (\mathrm{d} \theta / 2) \approx \mathrm{d} \theta / 2$.

$$
\left(\sigma_{r} h-\sigma_{\theta} h+2 \tau r\right) \mathrm{d} r+r h \mathrm{~d} \sigma_{r}=0
$$

If we assume that the composite particle is an ideal plastic solid and that the frictional condition between the composite particle and the bead is described by

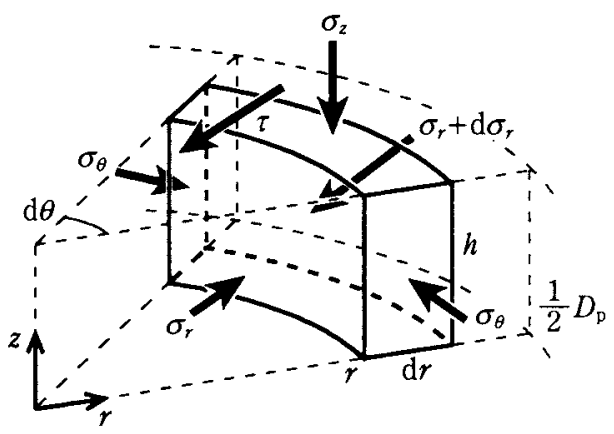

Fig. 3 Stresses acting on disk-shaped particles using a constant coefficient of Coulomb friction without sliding, the relation $\sigma_{\theta}=\sigma_{r}$ holds from the LevyMises equation. ${ }^{4}$ The shearing stress $\tau$ is expressed by Eq. (16) from von Mises' yield criterion. ${ }^{4}$

$$
\tau=-\sigma_{0} / \sqrt{3}
$$

$\sigma_{0}$ represents the yield stress of a composite particle. The following equation is obtained by substituting Eq. (16) into Eq. (15).

$$
\frac{d \sigma_{r}}{d r}-\frac{2 \sigma_{0}}{\sqrt{3} h}=0
$$

By disregarding the effects of shearing stress at the edge of a composite particle on plastic deformation, $\sigma_{r}$ is expressed by

$$
\sigma_{r}=\sigma_{0}-p
$$

where $p$ indicates the stress required to cause plastic deformation. The following equation is obtained by solving Eq. (17) (after substitution of Eq. (18)) with the boundary condition $p=\sigma_{0}$ at $r=D_{\mathrm{p}} / 2$.

$$
p=\sigma_{0}\left[1+\frac{2}{\sqrt{3} h}\left(\frac{D_{\mathrm{p}}}{2}-r\right)\right]
$$

The average $\bar{p}$ of the stress $p$ is given by Eq. (20).

$$
\bar{p}=\frac{\int_{0}^{D_{\mathrm{p}} / 2} 2 \pi p r \mathrm{~d} r}{\pi D_{\mathrm{p}}^{2} / 4}=\sigma_{0}\left(1+\frac{D_{\mathrm{p}}}{3 \sqrt{3} h}\right)
$$

$P_{\mathrm{pl}}\left(D_{\mathrm{p}}\right)$ is defined as a function whose value decreases as diameter $D_{\mathrm{p}}$ of a composite particle increases because of a linear relation between $\bar{p}$ and $D_{\mathrm{p}}$ as indicated by Eq. (20).

$$
P_{\mathrm{pl}}\left(D_{\mathrm{p}}\right)=k D_{\mathrm{p}}^{-\mathrm{g}\left(C_{\mathrm{WC}}\right)}
$$

$k$ is a constant that is dependent on physical properties of materials. $g\left(C_{\mathrm{WC}}\right)$ represents the magnitude of the effect of ceramic powder concentration $C_{\mathrm{WC}}$ in the composite particle on plastic deformation. The following equation is obtained by substituting Eq. (20) into Eq. (21).

$$
P_{\mathrm{pl}}\left(D_{\mathrm{p}}\right)=k\left[3 \sqrt{3} h\left(\frac{\bar{p}}{\sigma_{0}}-1\right)\right]^{-g\left(C_{\mathrm{WC}}\right)}
$$

$P_{\mathrm{pl}}\left(D_{\mathrm{p}}\right)$ is calculated with Eq. (22) by assuming that the impact force between a pair of beads is equivalent to the centrifugal force generated by the rotational motion of the rotor.

Both the elastic modulus and strength of fine particle-dispersed metal material are proportional to the total volume of fine particles in the material. The movement of the dislocation line in the material, 
which is necessary for causing plastic deformation, becomes more difficult as the amount of fine particles increases. ${ }^{5}$ Accordingly, a simple formula is used to express $g\left(C_{\mathrm{WC}}\right)$.

$$
g\left(C_{\mathrm{WC}}\right)=c_{1}+c_{2} \cdot C_{\mathrm{WC}}
$$

$c_{1}$ and $c_{2}$ are constants that depend on the physical properties of materials.

\section{Experimental and calculation methods}

The appropriate amount of the mixture of gas-atomized $\mathrm{Ag}-\mathrm{Ni}$ alloy particles (Ni content $3.2 \mathrm{wt} \%$, median diameter $7 \mu \mathrm{m}$, true density $1.05 \times 10^{4} \mathrm{~kg} / \mathrm{m}^{3}$ ) and WC fine powder (median diameter $0.7 \mu \mathrm{m}$, true density $1.56 \times 10^{4} \mathrm{~kg} / \mathrm{m}^{3}$ ) was charged together with a medium (zirconia beads) into a high-speed ellipticalrotor-type powder mixer. ${ }^{6}$ The charge volume ratio $f$ (i.e., apparent volume of the powder-bead mixture divided by the effective volume of the vessel) was 0.2 . The rotational speed of the vessel was maintained at $1.67 \mathrm{~s}^{-1}$, and rotor speed $N_{\mathrm{r}}$ was set at $25.0-41.7 \mathrm{~s}^{-1}$. The volume ratio $V_{\mathrm{p}} / V_{\mathrm{b}}$ of the powders to the beads was varied within the range of $0.3-1.5$, the concentration $C_{\mathrm{WC}}$ of WC in the composite particles within the range of $1.0-10 \mathrm{wt} \%$, and the size $D_{\mathrm{b}}$ of the beads in the range of $0.3-1.0 \mathrm{~mm}$. An image analyzer mounted on a metallurgical microscope was used to measure more than 5,000 particles to determine the size distributions (area basis) of composite particles.

The constants $k, c_{1}$ and $c_{2}$, which were necessary to calculate size distribution, were determined $(k=5.00 \times$ $10^{4}, c_{1}=14.2$ and $c_{2}=3.54 \times 10^{-2}$ ) from the particle compounding experiments under the several conditions ${ }^{2}$. Calculations were based on the assumptions that WC powder was dispersed uniformly in the powder mixture at the processing time $t=0$ and that the yield stress of composite particles was dependent only on the WC concentration.

\section{Results and discussion}

\subsection{Size distribution of composite particles}

Figure 4 shows a typical example of calculation and experimental results for temporal change in the size distribution of composite particles. In the early stage of processing, the particle growth phenomenon caused by the plastic deformation of particles was highly evident. Particle growth reached the equilibrium state as processing progressed owing to the balance between the forces necessary for causing plastic deformation and acting on the composite particles.

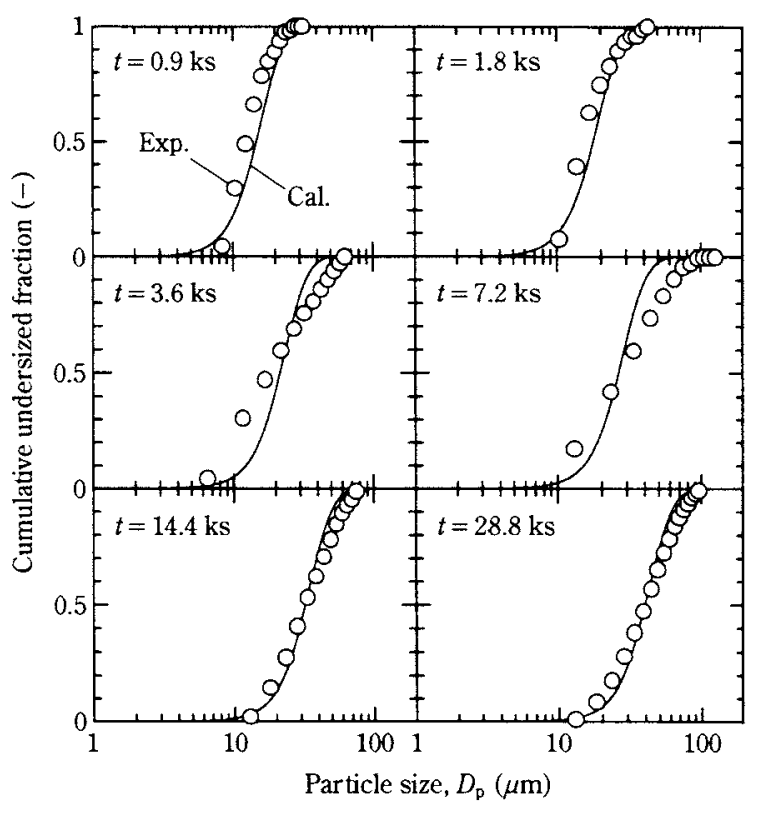

Fig. 4 Change in size distribution curves with the passage of processing time $t\left(C_{\mathrm{WC}}=10 \mathrm{wt} \%, D_{\mathrm{b}}=1.0 \mathrm{~mm}, N_{\mathrm{r}}=41.7 \mathrm{~s}^{-1}\right.$, $\left.V_{\mathrm{p}} / V_{\mathrm{b}}=0.3\right)$

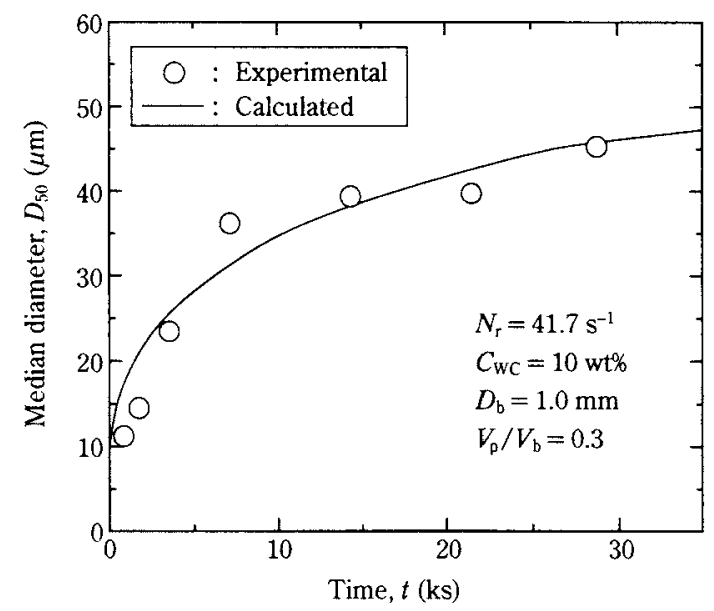

Fig. 5 Change in $D_{50}$ with the passage of processing time $t$

Figure 5 shows the temporal change in median diameter $D_{50}$ obtained from the data in Figure 4. Calculation results coincided closely with experimental results. In the following section, the validity of the proposed model is verified by comparing calculated values with the experimental data for $D_{50}$.

\subsection{Effects of the compounding conditions}

Figure 6 shows the relation between the median diameter $D_{50}$ of composite particles and the processing time $t$ when varying the powder-bead volume ratio $V_{\mathrm{p}} / V_{\mathrm{b}}$. In calculations and experimental results the particle growth rate decreased as $V_{p} / V_{b}$ increased 


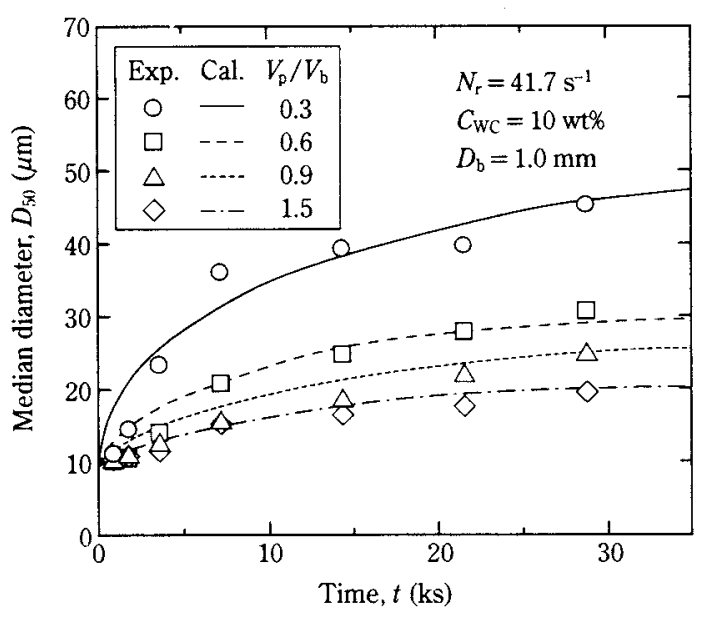

Fig. 6 Effect of volume ratio $V_{\mathrm{p}} / V_{\mathrm{b}}$ on temporal change in $D_{50}$

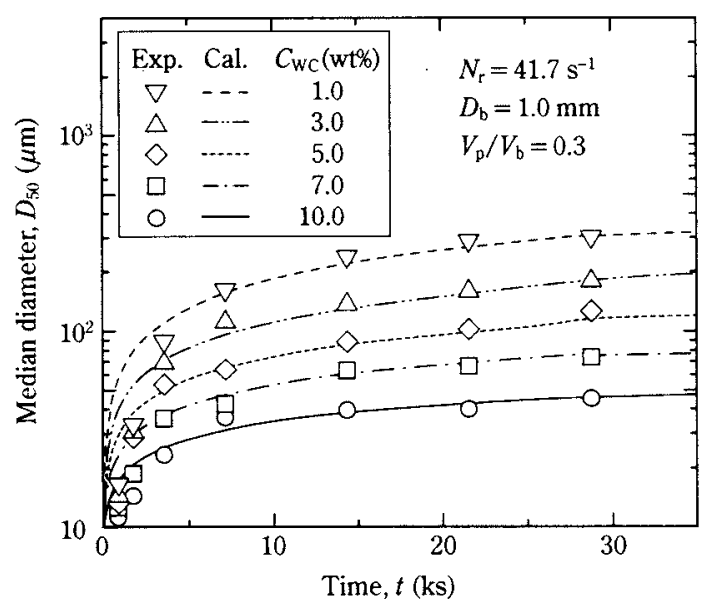

Fig. 7 Effect of WC concentration $C_{W C}$ on temporal change in $D_{50}$

because of the decreasing probability that composite particles were held by beads.

Figure 7 shows the effect of WC powder weight concentration $C_{\mathrm{WC}}$ on the temporal change in $D_{50}$. The size of composite particles in the late stage of processing declined with the increase in $C_{\mathrm{WC}}$. This means that the presence of WC powder in composite particles prevented the composite particles from causing the plastic deformation that is necessary for growth.

Figure $\mathbf{8}$ shows the effect of rotor speed $N_{\mathrm{r}}$ on particle growth. At higher $N_{\mathrm{r}}$, the particle growth rate increased due to the increase in the frequency per unit time with which the mixture of composite particles and beads was compressed by the rotor at the minimum clearance. However, comparing the results in Figure $\mathbf{8}$ with those in Figure $\mathbf{6}$ shows that the effect of $N_{\mathrm{r}}$ on particle growth was smaller than that of $V_{\mathrm{p}} / V_{\mathrm{b}}$. At higher rotor speeds, particle growth occurs only with difficulty even though the compres-

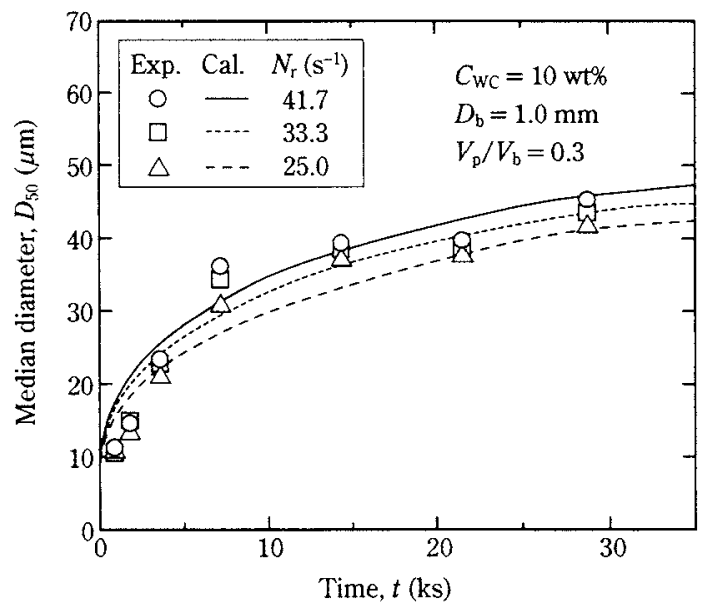

Fig. 8 Effect of rotor speed $N_{\mathrm{r}}$ on temporal change in $D_{50}$

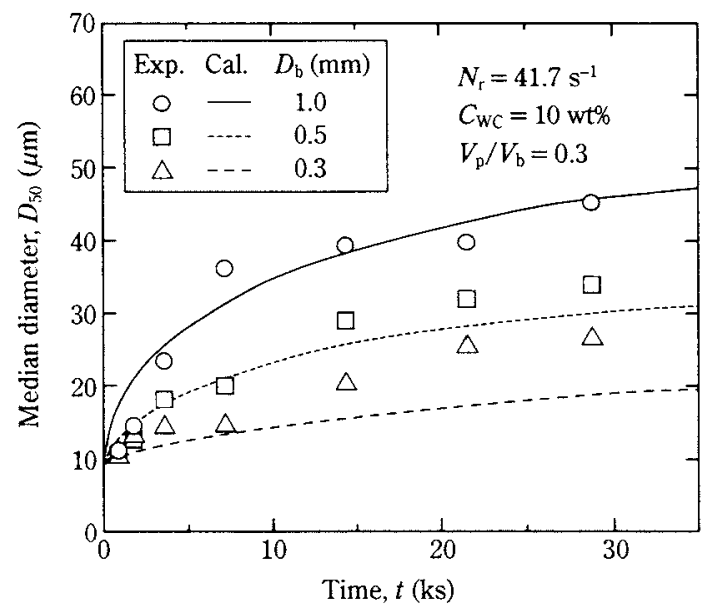

Fig. 9 Effect of bead diameter $D_{\mathrm{b}}$ on temporal change in $D_{50}$

sion frequency increases more if the amount of beads is insufficient to cause plastic deformation.

Figure 9 shows the calculation and experimental results obtained when bead diameter $D_{\mathrm{b}}$ was varied. Composite particle size decreased when using smallersized beads because of change in the stress acting on composite particles and in the probability of holding a composite particle. ${ }^{?}$

Close agreement between calculated values and experimental data for particle size in Figures 6 through 9 supports the validity of the proposed model.

\section{Conclusion}

We performed a kinetic analysis, and investigated the mechanism, of a composite particle growth phenomenon brought about by plastic deformation of composite particles occurring in a compounding process involving the uniform dispersion of fine 
ceramic powder into alloy particles. The following conclusions can be drawn.

1) Particle growth in the compounding process has been conceived as a probability phenomenon in which composite particles are caught between a pair of media and subjected to plastic deformation. A simplified model of the phenomenon has been proposed in terms of the probability function of composite particle growth. The validity of the model has been confirmed by agreement between calculated values and experimental data.

2) Both the calculations and the experiments clarified the effects of compounding conditions, i.e., the powder-media volume ratio, the amount of ceramic powder added, rotor speed, and medium diameter, on particle growth.

\section{Nomenclature}

A : projected area of composite particle

$\left(\mathrm{m}^{2}\right)$

$A_{\mathrm{s}} \quad$ : projected area of spherical particle having volume equal to that of composite particle

$b \quad:$ constant expressed by Eq. (8)

$C_{\mathrm{WC}} \quad$ : weight concentration of WC in composite particles

$c_{1}, c_{2} \quad$ : constants in Eq. (23)

$D_{50}$ : area median diameter of composite particle

$D_{\mathrm{b}} \quad$ : bead diameter

$D_{\mathrm{p}} \quad$ : disk-shaped particle diameter

$F \quad$ : impact force between a pair of beads

$f \quad$ : charge volume ratio

$f\left(D_{\mathrm{p}}, t\right)$ : frequency distribution function

$\left(\mathrm{m}^{-1}\right)$

$g\left(C_{\mathrm{WC}}\right)$ : function of $C_{\mathrm{WC}}$ defined by Eq. (23)

$h \quad$ : height of disk-shaped particle

$k \quad$ : constant in Eq. (21)

$N_{\mathrm{r}}, N_{\mathrm{v}}$ : rotational speeds of rotor and vessel $\quad\left(\mathrm{s}^{-1}\right)$

$P_{\mathrm{n}}\left(D_{\mathrm{p}}\right)$ : probability function expressed by Eq. (13)

$P_{\mathrm{pl}}\left(D_{\mathrm{p}}\right)$ : probability function defined by Eq. (21) (-)

$P_{z}\left(D_{\mathrm{p}}\right)$ : probability function defined by Eq. (4) (-)

$p \quad$ : stress required to cause plastic deformation

$S_{\text {in }}, S_{\text {out }}:$ projected areas of composite particles expressed by Eqs. (2) and (3)
$S(t) \quad$ : total projected area of composite particles

$t \quad$ : processing time

$\begin{array}{lll}V_{\mathrm{a}} & : \text { apparent volume of powder mixture } & \left(\mathrm{m}^{3}\right) \\ V_{\mathrm{b}} & : \text { apparent charge volume of beads } & \left(\mathrm{m}^{3}\right)\end{array}$

$V_{\mathrm{m}} \quad$ : effective volume of mixer vessel $\quad\left(\mathrm{m}^{3}\right)$

$V_{\mathrm{p}} \quad$ : apparent charge volume of powder mixture

$v_{\mathrm{a}} \quad$ : apparent volume of composite particles present in voids among beads $\quad\left(\mathrm{m}^{3}\right)$

$Z \quad$ : frequency expressed by Eq. (5) (-)

$\alpha \quad$ : angle representing contact point between composite particle and bead (rad)

$\gamma \quad$ : area ratio defined by Eq. (11)

$\mu \quad$ : coefficient of dynamic friction between composite particle and bead $\quad(-)$

$\sigma_{0} \quad$ : yield stress of composite particle $\quad(\mathrm{Pa})$

$\sigma_{r}, \sigma_{\theta}$ : stresses applied in directions of $r$ and $\theta(\mathrm{Pa})$

$\tau \quad$ : shearing stress $\quad(\mathrm{Pa})$

$\phi \quad$ : shape factor defined by Eq. (14) $\quad(-)$

\section{References}

1) Satoh, M., T. Yanagida and T. Iwasaki, "Dispersion and Compounding Process of Particulate Ag-Ni Alloy and Fine WC Powder Using a High-speed Elliptical-rotortype Powder Mill," J. Jpn Soc. Powder and Powder Met., 44, 618-621 (1997).

2) Iwasaki, T., T. Yanagida and M. Satoh, "Effect of Operating Conditions in Powder Metal Compounding Process on Particle Size Change," J. Jpn Soc. Powder and Powder Met., 45, 271-275 (1998).

3) Kuwahara, Y., F. Saito and S. Yashima, "An Analysis of Selection Function of Vibration Mill," J. Res. Ass. Powder Technol., Japan, 14, 319-325 (1977).

4) Dieter, G. E., Mechanical Metallurgy, 3rd ed., McGrawHill, Inc. (1986).

5) Fukuda, H., R. Yokota and I. Shiota, Fukugou Zairyou Kisokougaku, Nikkan Kougyo (1994).

6) Satoh, M., T. Yoshida, K. Miyanami and Y. Okudaira, "Evaluation of the Performance of a High-speed Elliptical-rotor-type Powder Mixer," J. Soc. Powder Technol., Japan, 31, 789-794 (1994).

7) Yamauchi, K, Y. H. Park, H. Hashimoto and R. Watanabe, "Change in Morphology and Some Properties of Ti-Al Powder Particles during Mechanical Alloying by Vibratory Ball Milling," J. Jpn Soc. Powder and Powder Met., 38, 42-46 (1991). 


\section{Author's short biography}

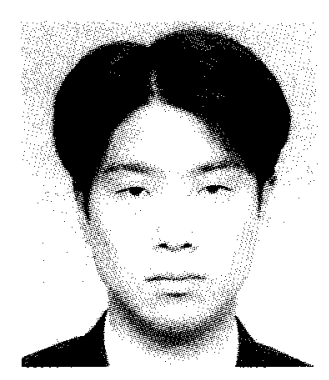

\section{Tomohiro Iwasaki}

Tomohiro Iwasaki received the master degree in chemical engineering from the Osaka Prefecture University in 1996. He joined the Department of Chemical Engineering, the Osaka Prefecture University, as an Assistant Professor in 1997. His major research interests are design of functional composite particle, evaluation of manufacturing process of composite particles, simulation of behavior of particle in compounding process, measurement and evaluation of dynamic physical properties of powder, and analysis of stress in powder bed. 